\title{
New measures of wind angular dispersion in three dimensions
}

\author{
P. S. Farrugia \& A. Micallef \\ Department of Physics, Faculty of Science, University of Malta, Malta
}

\begin{abstract}
Wind is a $3 \mathrm{D}$ vector quantity. However, it is frequently found that the vertical component is much smaller in magnitude than the horizontal ones and acts over shorter distances so that its effect is considered negligible. As a result of this, wind is often treated as a two dimensional circular variable. In fact, most of the statistical treatment used to derive the wind angular dispersion, a parameter that is very important in determining the rate of diffusion of pollutants, is based on this assumption. Nevertheless, the reduction of a dimension might not always be justified. A case in point occurs when considering air flow in a street canyon whereby all three directions of air flow can be significant. In such a situation, a 3D measure of angular dispersion would be much more appropriate. For this reason, this work concentrates on establishing consistent $3 \mathrm{D}$ measures of angular dispersion. Thus a detailed geometric analysis of an existing and widely used measure of 3D angular dispersion was carried out. This showed that this measure accounts for dispersion mainly along one direction. In order to improve on this, a new set of measures for angular dispersion that account for all three directions is derived using some recently proposed measures of angular dispersion. It is also shown that these new measures can be further extended to any dimension.
\end{abstract}

Keywords: spherical variables, three dimensional measure of angular dispersion, spherical variance, projection variance, geometric variance, diffusion of pollutants in three dimensions, angular dispersion in any dimension.

\section{Introduction}

Air motion, can occur in any of the three spatial dimensions. However, it is common practice to ignore the vertical component since this is frequently much 
smaller in magnitude than the other two and acts over shorter distances. This procedure has the effect of reducing wind from a spherical to a circular variable.

One of the consequences of this reduction in the number of dimensions is that the angular standard deviation is calculated using equations from circular statistics. These can be either the simple ones proposed by Mardia [1] and Batschelet [2] that were obtained by linearization of the angles using some type of mapping, or else, more often, by employing complex, multi-step algorithms like those of Essenwanger [3], Irwin [4], Nelson [5], Skibin [6] and Yamartino [7]. Once the result is obtained, it is then employed to determine the rate of diffusion of pollutants. The important thing to note is that the resultant pollutant diffusion rate applies only to the horizontal plane in which the $x$-and $y$-axis lie and gives no information about the vertical diffusion.

A more complete analysis would show that even though the procedure of limiting the number of dimensions that are considered is often justified, it cannot be applied indiscriminately in the general case. A very important example where this approximation is not appropriate is provided by air motion in a street canyon whereby all three components can be significant due to the vortices that can be induced by the vertical walls. Another case arises in connection with the emission of a hot plume, such as the exhaust from motor vehicles, where the buoyancy effect can make the vertical component of air motion sizeable. For such situations, a three dimensional measure of angular dispersion would seem a more appropriate parameter to use in order to calculate the diffusion of air pollutants.

Such considerations lead to the need to investigate 3D measures of dispersion that can be used in 3D pollutant dispersion models. To the knowledge of the authors, the only 3D measure of dispersion available so far in the literature is the spherical variance given by Mardia [1],

$$
s_{\text {Mardia } 3}^{2}=1-R_{3}
$$

where $R_{3}$ is the magnitude of the vector to the centre of gravity of the $3 \mathrm{D}$ system $\mathbf{R}_{3}$ (see eqn (15)). This equation is a generalisation of the circular variance that Mardia [1] proposed in the case of circular variables. However, this extension to a higher number of dimensions is not straight forward, and no indication was foreseen in the work of how the derivation used for the circular variables can be extended directly to the $3 \mathrm{D}$ case. Justification for doing so comes from the fact that the equation has the same form as that of circular variance and that $R_{3}$ is a measure of the concentration of the angles. In fact for a unimodal distribution the values of $R_{3}$ range from zero (when the angles are highly dispersed) to one (when all the angles in the sample are along one single direction).

The problem encountered by Mardia [1] to extend his measure of angular dispersion to higher dimensions is also encountered in the case of other equations that have been proposed for the angular variance. In fact, looking at the other algorithms and the way in which they were derived, there does not seem to be an intuitive way of including an additional angle and proceed to derive a consistent measure of angular dispersion. This means that the transition from circular to spherical variables for these equations is not straight forward 
even though circular variables should, in theory, be a subset of spherical variables.

An alternative pathway is now possible through the geometric approach taken by Farrugia and Micallef [8]. In this work, using the fact that in general the most important thing is the distance of the angle from the average angle of the sample $\theta_{\mathrm{a}}$ (see eqn (6)), a suitable line on which to map the angles or their difference from $\theta_{\mathrm{a}}$ was chosen. This led to the development of two new measures of 2D angular dispersion, termed the projection variance,

$$
s_{\mathrm{P} 2}^{2}=1-R_{2}^{2}-s_{\mathrm{S}^{\prime}}^{2}
$$

and the geometric variance,

$$
s_{\mathrm{G} 2}^{2}=2\left(1-R_{2}\right)-s_{\mathrm{S}^{\prime}}^{2}
$$

where $R_{2}$ is the magnitude of the vector to the centre of gravity of the system in the $2 \mathrm{D}$ case $\mathbf{R}_{2}$ (see eqn (8)), while $s_{\mathrm{S}^{\prime}}^{2}$ gives the variance of the sines of the angles in a direction rotated such that $\mathbf{R}_{2}$ is aligned with the $x^{\prime}$-axis (see eqn (11)).

The real important difference that stems out between these and the linear equations proposed by Mardia [1] (eqn (9)) and Batschelet [2] (eqn (10)) is the term $s_{\mathrm{S}^{\prime}}^{2}$. In the work by Farrugia and Micallef [8] it was shown that, from a geometric perspective, while $R_{2}$ gives a measure of angular concentration along the direction of $\mathbf{R}_{2}, s_{\mathrm{S}^{\prime}}^{2}$ gives a measure of angular concentration along a line perpendicular to $\mathbf{R}_{2}$. Thus $R_{2}$ and $s_{\mathrm{S}^{\prime}}^{2}$ together give a $2 \mathrm{D}$ picture of the concentration of the angles along two orthogonal directions. In this way, their negative will give a measure of dispersion in two 2D. Farrugia and Micallef [8] also showed that $s_{\mathrm{S}^{\prime}}^{2}$ emerges naturally if the Taylor or Fourier expansions are used to calculate the variance of the sample angles.

Further analyses on circular distributions carried out in the course of the work presented here reveals that $s_{\mathrm{S}^{\prime}}^{2}$ is also one of the terms that can emerge when calculating the angular variance of standard distributions. For example, the angular variance of the von Mises distribution with mean zero and concentration parameter $\kappa$ obtained by Fisher [9], can be written in the form,

$$
\operatorname{var}[\theta]=\left(\frac{\pi^{2}}{3}+1\right)-4 \rho-2 \sigma_{\mathrm{S}^{\prime}}^{2}+4 \sum_{n=3}^{\infty} \frac{(-1)^{n} I_{n}(\kappa)}{I_{0}(\kappa)}
$$

where $I_{n}(\kappa)$ is a modified Bessel function of the first kind and order $n$, while $\rho$ and $\sigma_{\mathrm{S}^{\prime}}^{2}$ are the population parameters corresponding to $R_{2}$ and $s_{\mathrm{S}^{\prime}}^{2}$. In a similar fashion, using the Fourier expansion of the wrapped normal distribution [1] and that for the minimum angular distance squared $[8,9]$ it is a matter of going through the algebra to show that for this distribution the variance is given by,

$$
\operatorname{var}[\theta]=\left(\frac{\pi^{2}}{3}+1\right)-4 \rho-2 \sigma_{\mathrm{S}^{\prime}}^{2}+4 \sum_{i=3}^{\infty} \frac{(-1)^{i} \rho^{\left(i^{2}\right)}}{i^{2}}
$$


Additionally, in these particular cases, the first few terms of eqns (4) and (5) are actually the same, even though the distributions are different. This gives further indication of the need for using both $R_{2}$ and $s_{\mathrm{S}^{\prime}}^{2}$ in order to obtain a consistent two dimensional measure of angular dispersion.

Another important feature of the projection and geometric variance is that the $2 \mathrm{D}$ derivation can be extended to three and higher order dimensions. This is the main scope of this work. In the process it will also be shown that the expression in $k$-dimensions contains $k$ terms that can be identified as measure of dispersion in $k$ orthogonal directions. However, before proceeding any further, in the next section, some important two dimensional parameters are defined.

\section{Some important two dimensional parameters}

In this section some important 2D parameters are defined. The first one is the mean angle of the sample $\theta_{\mathrm{a}}$, which for a sample of angles $\theta_{i}$ of size $n$ is defined as,

$$
\theta_{\mathrm{a}}=\tan ^{-1}(\bar{S} / \bar{C})
$$

where $\bar{S}$ and $\bar{C}$ are the averages of the sines and the cosines of the angles respectively i.e.,

$$
\bar{S}=\frac{1}{n} \sum_{i=1}^{n} \sin \left(\theta_{i}\right) \text { and } \bar{C}=\frac{1}{n} \sum_{i=1}^{n} \cos \left(\theta_{i}\right) .
$$

Note that when evaluating $\theta_{\mathrm{a}}$, care needs to the taken of the quadrant in which $\bar{S}$ and $\bar{C}$ will locate the angle.

This definition for the mean angle of the sample was obtained by mapping each angle $\theta_{i}$ to the unit vector ${ }_{2} \hat{\mathbf{r}}_{i}=\left[\sin \left(\theta_{i}\right), \cos \left(\theta_{i}\right)\right]$. Subsequently, $\theta_{\mathrm{a}}$ was derived as the angle that the mean of the vectors $\mathbf{R}_{2}$, termed the vector towards the centre of mass of the system, makes with the positive $x$-axis.

The magnitude or length of $\mathbf{R}_{2}$, given by,

$$
R_{2}=\sqrt{\bar{S}^{2}+\bar{C}^{2}}
$$

is another important parameter. Its significance stems from the fact that for a unimodal distribution, its value gives an indication of the dispersion of the angles along a single direction. In fact its value ranges from zero (when the angles exhibit extensive dispersion) to one (when all the angles are pointing in the same direction). For this reason, $-R$ is often taken as a measure of dispersion, as can be seen by looking at the equations used as measures of angular dispersion such as the circular variance given by Mardia [1],

$$
s_{\text {Mardia } 2}^{2}=1-R_{2}
$$

and angular variance proposed by Batschelet [2],

$$
s_{\text {Bat }}^{2}=2\left(1-R_{2}\right)
$$


Consider a rotation of the coordinate system by an angle $\theta_{\mathrm{a}}$ such that in the new $x^{\prime} y^{\prime}$ coordinate system the new mean angle of the sample $\theta_{\mathrm{a}}^{\prime}=0$. In such a system $\mathbf{R}_{2}$ is aligned along the $x^{\prime}$ direction, so that $R_{2}$ becomes a measure of dispersion along this direction.

However, provided that not all the angles are aligned in the same direction, then there should also be some "concentration" about the $y^{\prime}$ direction and as so it should be possible to find some measure of concentration along this direction. This can be provided by the variance of the sines along the $y^{\prime}$-axis [8],

$$
s_{\mathrm{S}^{\prime}}^{2}=\frac{1}{n} \sum_{i=1}^{n} \sin ^{2}\left(\theta_{i}-\theta_{\mathrm{a}}\right)
$$

(Note that the biased form of the variance is being adopted in order to simplify the equations derived.) The reason for this is that $s_{\mathrm{S}^{\prime}}^{2}$ takes values ranging from zero, when all the angles are aligned along the $x^{\prime}$-axis, to one, when all the angles are aligned along the $y^{\prime}$-axis. Naturally, the upper limit cannot be attained because of the alignment of $\mathbf{R}_{2}$ with the $x^{\prime}$-axis. Thus, in a similar way as was done for $R_{2}$, it is possible to consider $s_{\mathrm{S}^{\prime}}^{2}$ as a measure of dispersion along the $y^{\prime}$ direction.

It can be seen that in $2 \mathrm{D}$ it is possible to find two parameters that represent measures of concentration along two orthogonal directions so that their negatives can be taken as measures of dispersion along these same directions. In the next section it will be shown how it is possible to obtain three parameters of concentration/dispersion for spherical variables.

\section{Derivation of measures of dispersion in three dimensions}

In this section the derivations of the projection and geometric variance as given in [8] will be generalised to $3 \mathrm{D}$. As in the $2 \mathrm{D}$ case, the first thing that needs to be done is to find a suitable geometric measure, by which it is meant that a line will be found such that the projections on this line can be representative of the $3 \mathrm{D}$ system.

Thus, given a sample $\left(\phi_{i}, \theta_{i}\right)$ of 3D wind directions (see fig. 1), these can be mapped on the unit vector,

$$
{ }_{3} \hat{\mathbf{r}}_{i}=\left(\begin{array}{c}
X_{i} \\
Y_{i} \\
Z_{i}
\end{array}\right)=\left[\begin{array}{c}
\sin \left(\theta_{i}\right) \cos \left(\phi_{i}\right) \\
\sin \left(\theta_{i}\right) \sin \left(\phi_{i}\right) \\
\cos \left(\theta_{i}\right)
\end{array}\right]
$$

At this point, since the general form of the components will always be the same in any coordinate system, without loss of generality, the projections on the $x, y$ and $z$-axis will be analysed to assess the potential use of each coordinate axis as the line that represents the angles. Once an adequate axis is chosen, then it is possible to rotate the coordinate system so as to optimise the orientation of the axis. 


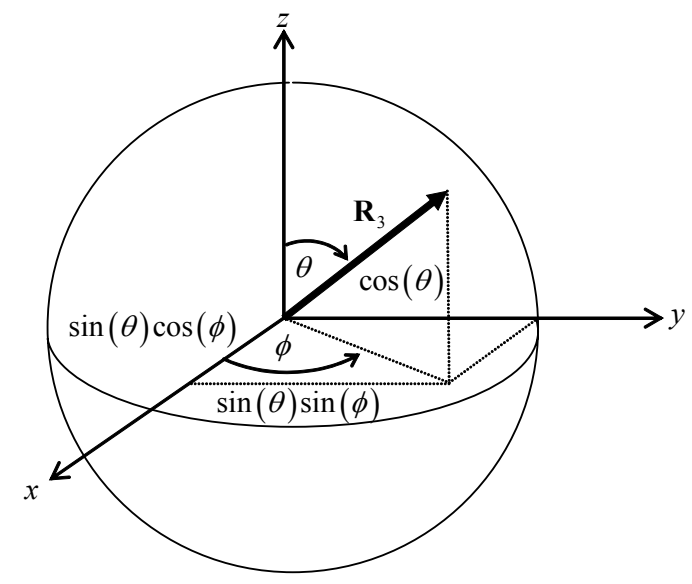

Figure 1: $\quad$ The spherical coordinate system representation in 3D.

For an axis to be able to represent adequately all three special directions, it is necessary that the component along it contains terms with both $\phi_{i}$ and $\theta_{i}$. This means that the $z$-component cannot be taken. As for the other two axes, in order to discriminate between them, use can be made of the same criteria that has been adopted in the two-dimensional case i.e. that the cosine term increases monotonically when the angle is in the range $[0, \pi]$, while the sine term increases monotonically only when the angle is in the range $[0,0.5 \pi]$. Thus, mapping on the $x$-axis will retain much more information than mapping on the $y$-axis. Hence, it can be concluded that the $x$-axis is the most suitable candidate for the geometric measure.

Even though ideally all terms should involve cosines so that they retain maximum information, given the way in which $\phi_{i}$ and $\theta_{i}$ are defined this is not possible since $\cos \left(\theta_{i}\right) \cos \left(\phi_{i}\right)$ is not a component along any fixed line.

At this stage the question arises weather it makes sense to take as the geometric measure $1-X_{i}\left(=1-\sin \left(\theta_{i}\right) \cos \left(\phi_{i}\right)\right)$ as was done in the $2 \mathrm{D}$ case, or whether $X_{i}$ should be used. In response to this question it is worth noting that to develop the geometric measure in $3 \mathrm{D}$, before mapping the angles on a line, they are being projected on a circle (through the term $\sin (\theta)$ ). Once the sphere has been projected on the circle, the argument used in the 2D case [8] can be adopted. One may recall that the argument used was that for the unit circle, the arc length is equal to the subtended angle measured in radiance and hence given that $1-\cos (\theta)$ is a good measure of the arch length then it is also a good measure of the angle. It follows that $1-X_{i}$ should be used.

Once the appropriate axis and measure have been established, the coordinate system can be rotated so as to optimise its orientation. The adopted criterion is once again similar to the one used in the $2 \mathrm{D}$ case, i.e. an orientation will be chosen such that it minimises the expected mean direction. Thus we need to minimise, 


$$
\frac{1}{n} \sum_{i=1}^{n}\left[1-\sin \left(\theta_{i}-\beta\right) \cos \left(\phi_{i}-\alpha\right)\right]
$$

with respect to both $\alpha$ and $\beta$. This can be done by differentiating with respect to both $\alpha$ and $\beta$, setting the results equal to zero,

$$
\frac{1}{n} \sum_{i=1}^{n} \cos \left(\theta_{i}-\beta\right) \cos \left(\phi_{i}-\alpha\right)=0 \text { and } \frac{1}{n} \sum_{i=1}^{n} \sin \left(\theta_{i}-\beta\right) \sin \left(\phi_{i}-\alpha\right)=0
$$

and solving the two simultaneous equations. To solve this system of equations, consider the vector to the centre of gravity in three dimensions $\mathbf{R}_{3}$. If the coordinate system is rotated such that $\mathbf{R}_{3}$ is aligned with the new $x^{\prime}$-axis, $\mathbf{R}_{3}$ can be written simply as,

$$
R_{3}=\frac{1}{n} \sum_{i=1}^{n} \sin \left(\theta_{i}-\theta_{\mathrm{a}}\right) \cos \left(\phi_{i}-\phi_{\mathrm{a}}\right),
$$

where $\theta_{\mathrm{a}}$ and $\phi_{\mathrm{a}}$ give the mean direction $[1,10]$. If both sides of eqn (15) are differentiated with respect to $\theta_{\mathrm{a}}$ and $\phi_{\mathrm{a}}$, remembering that $R_{3}$ is a constant, then eqns (14) would be recovered if $\alpha$ and $\beta$ are equated to $\phi_{\mathrm{a}}$ and $\theta_{\mathrm{a}}$. This means that the geometric measure is optimised if the $x$-axis is aligned with $\mathbf{R}_{3}$.

Note that aligning the $x$-axis with $\mathbf{R}_{3}$ would not minimise all the possible moments of any distribution. In order to obtain this, the distribution from which the sample has been taken needs to be highly symmetric and $n$ has to be large.

Once the geometric measure has been established, the projection variance can be obtained by equating the system of angles $\left(\phi_{i}, \theta_{i}\right)$ to the corresponding $1-X_{i}^{\prime}$ $\left(=1-\sin \left(\theta_{i}-\theta_{\mathrm{a}}\right) \cos \left(\phi_{i}-\phi_{\mathrm{a}}\right)\right)$ and then calculating the variance,

$$
s_{\mathrm{P} 3}^{2}=\frac{1}{n} \sum_{i=1}^{n} X_{i}^{\prime}-\left\{\frac{1}{n} \sum_{i=1}^{n} X_{i}^{\prime}\right\}^{2},
$$

where in deriving this expression use has been made of the identity $\operatorname{var}[a x+b]=$ $a^{2} \operatorname{var}[x]$, with $a$ and $b$ being constants. On the other hand the 3D geometric variance can be obtained by equating the system of angles $\left(\phi_{i}-\phi_{\mathrm{a}}, \theta_{l}-\theta_{\mathrm{a}}\right)$ with $1-$ $X_{i}^{\prime}$. In this case the resulting equation would be,

$$
s_{\mathrm{G} 3}^{2}=\frac{1}{n} \sum_{i=1}^{n}\left[1-\sin \left(\theta_{i}-\theta_{\mathrm{a}}\right) \cos \left(\phi_{i}-\phi_{\mathrm{a}}\right)\right]^{2} .
$$

After some mathematical manipulation using trigonometric relations, it is possible to rewrite eqns (16) and (17) as,

$$
s_{\mathrm{P} 3}^{2}=1-R_{3}^{2}-s_{y^{\prime}}^{2}-s_{z^{\prime}}^{2} \text { and } s_{\mathrm{G} 3}^{2}=2\left(1-R_{3}\right)-s_{y^{\prime}}^{2}-s_{z^{\prime}}^{2} .
$$

where $s_{y^{\prime}}^{2}$ and $s_{z^{\prime}}^{2}$ are the variances of the components of the system of angles in the $x^{\prime} y^{\prime} z^{\prime}$ coordinate system:

$$
s_{y^{\prime}}^{2}=\frac{1}{n} \sum_{i=1}^{n} \sin ^{2}\left(\theta-\theta_{\mathrm{a}}\right) \sin ^{2}\left(\phi-\phi_{\mathrm{a}}\right) \text { and } s_{z^{\prime}}^{2}=\frac{1}{n} \sum_{i=1}^{n} \cos ^{2}\left(\theta_{i}-\theta_{\mathrm{a}}\right)
$$


In analogy with the $2 \mathrm{D}$ case the terms $R_{3}$ and $s_{y^{\prime}}^{2}$ can be easily identified with the measures of concentration along the $x^{\prime}$ and $y^{\prime}$-axis that have been identified for the circular variables, namely $R_{2}$ and $s_{\mathrm{S}^{\prime}}^{2}$ respectively. Hence their negative constitutes a measure of angular dispersion in the $x^{\prime} y^{\prime}$-plane. The really new term is $S_{z^{\prime}}^{2}$, which is again a measure of concentration, this time along the $z^{\prime}$-axis. Thus it needs to be subtracted from the angular variance. In this way the two measures of dispersion given by eqn (18) constitute an improvement over the measure of dispersion given by Mardia [1] for 3D spherical variables (eqn (1)). It can also be noted that the maximum value for the projection and geometric variance in the three-dimensional case is the same as for the circular case (one and two respectively for the biased form).

A further point that can be noted is that like in the 2D case [8] the mean bias (MB) of the geometric measure is equal to the mean absolute bias (MAB), given that $1-X_{i}^{\prime} \geq 0$ for any angle, and is equal to the spherical variance (eqn (1)):

$$
\mathrm{MB}=\mathrm{MAB}=\frac{1}{n} \sum_{i=1}^{n}\left[1-X_{i}^{\prime}\right]=1-R_{3} .
$$

This provides a direct derivation for the measure of dispersion proposed by Mardia [1]. However, in linear statistics, the mean bias and mean absolute bias are usually discarded in favour of the variance. Such a consideration confirms the superiority of the geometric or projection variance as compared to Mardia's spherical variance as a measure of $3 \mathrm{D}$ angular dispersion.

\section{Results for the $k$-dimensional case}

One of the most important aspects, at least from the academic point of view, of the derivation of the projection and geometric variance is that it can be extended to any dimension $k$. The steps to follow in the most general case parallel exactly those of the 3D case. Thus, taking a sample of angles in $k$-dimensions given by $\left(\theta_{1, i}, \theta_{2, i}, \theta_{3, i}, \ldots, \theta_{k-1, i}\right)$ this can be mapped to the unit vector,

$$
{ }_{k} \hat{\mathbf{r}}_{i}=\left(\begin{array}{l}
X_{1, i} \\
X_{2, i} \\
X_{3, i} \\
\vdots \\
X_{k, i}
\end{array}\right)=\left(\begin{array}{l}
\sin \left(\theta_{k-1, i}\right) \sin \left(\theta_{k-2, i}\right) \ldots \sin \left(\theta_{3, i}\right) \sin \left(\theta_{2, i}\right) \cos \left(\theta_{1, i}\right) \\
\sin \left(\theta_{k-1, i}\right) \sin \left(\theta_{k-2, i}\right) \ldots \sin \left(\theta_{3, i}\right) \sin \left(\theta_{2, i}\right) \sin \left(\theta_{1, i}\right) \\
\sin \left(\theta_{k-1, i}\right) \sin \left(\theta_{k-2, i}\right) \ldots \sin \left(\theta_{3, i}\right) \cos \left(\theta_{2, i}\right) \\
\vdots \\
\cos \left(\theta_{k-2, i}\right)
\end{array}\right) .
$$

Since it is desired that the derived geometric measure represents adequately all angles, the $x_{1}$-axis needs to be chosen. The geometric measure will then take the form $1-X_{1, i}$ since as in the 3D case, the $k$-spherical variable is being mapped successively onto a lower dimensional systems and when it is mapped on a circle the same criteria used for the 2D case will apply. 
The next thing to do is to orient the coordinate system so as to optimise the orientation. Using as a condition the need to minimise the moments, and focusing on the minimisation of the mean given by,

$$
\frac{1}{n} \sum_{i=1}^{n}\left[1-\sin \left(\theta_{k-1, i}-\alpha_{k-1}\right) \ldots \sin \left(\theta_{2, i}-\alpha_{2}\right) \cos \left(\theta_{1, i}-\alpha_{1}\right)\right],
$$

in a similar fashion to the $3 \mathrm{D}$ case, it can be determined that this occurs when $\alpha_{i}$ 's are equal to the $\theta_{\mathrm{a}, i}$ 's that give the direction of the vector towards the centre of gravity $\boldsymbol{R}_{k}$, in the $k$ dimensional system. Thus the resulting geometric measure would be given by, $1-X_{1, i}^{\prime}\left(=1-\sin \left(\theta_{k-1, i}-\theta_{\mathrm{a}, k-1}\right) \ldots \sin \left(\theta_{2, i}-\theta_{\mathrm{a}, 2}\right) \cos \left(\theta_{1, i}-\theta_{\mathrm{a}, 1}\right)\right)$

The projection variance can now be found by equating the set of angles $\left(\theta_{1, i}, \theta_{2, i}, \theta_{3, i}, \ldots, \theta_{k-1, i}\right)$ to the geometric measure and then determining the variance. The geometric variance would be given by equating $\left(\theta_{1, i}^{\prime}, \theta_{2, i}^{\prime}, \theta_{3, i}^{\prime}, \ldots, \theta_{k-1, i}^{\prime}\right)$ where, $\theta_{j, i}^{\prime}=\theta_{j, i}-\theta_{\mathrm{a}, i}$ to the geometric measure and then determining the variance. The final equations obtained using some trigonometric identities would be given respectively by,

$$
s_{\mathrm{P} k}^{2}=1-R_{k}^{2}-\sum_{j=2}^{k-1} s_{x_{j}^{\prime}}^{2} \text { and } s_{\mathrm{G} k}^{2}=2\left(1-R_{k}\right)-\sum_{j=2}^{k-1} s_{x_{j}^{\prime}}^{2},
$$

where $R_{k}=\left|\boldsymbol{R}_{k}\right|$, given by,

$$
R_{k}=\frac{1}{n} \sum_{i=1}^{n} \sin \left(\theta_{k-1, i}^{\prime}\right) \ldots \sin \left(\theta_{2 i}^{\prime}\right) \cos \left(\theta_{1 i}^{\prime}\right)=\frac{1}{n} \sum_{i=1}^{n} X_{1, i}^{\prime},
$$

and the $S_{x_{j}^{\prime}}^{2}$ 's are the variances of the components along the $x_{j}^{\prime}$-axis,

$$
s_{x_{j}^{\prime}}^{2}=\frac{1}{n} \sum_{i=1}^{n} X_{j, i}^{\prime 2} .
$$

Considering now the mean bias (MB) and the absolute bias (MAB) of this $k$ dimensional measure of dispersion, it can be easily shown that,

$$
\mathrm{MB}=\mathrm{MAB}=\frac{1}{n} \sum_{i=1}^{n}\left[1-X_{1, i}^{\prime}\right]=1-R_{k},
$$

which is Mardia's spherical variance for the $k$-dimensional system.

\section{Conclusion}

This work considers the need for consistent 3D measures of dispersion. The problematic issue considered here is that it is not straight forward to generalise the existing 2D measures of angular dispersion to include a higher dimension. An analysis of a known 3D measure of dispersion, that given by Mardia [1], reveals that while this measure has been obtained through a generalisation of Mardia's circular variance, it does not seem to have been justified through a direct generalisation of the derivation. 
In order to address the situation, two newly proposed measures of dispersion for circular variables, the projection and the geometric variance, have been generalised to three and higher dimensions. This has been accomplished by finding a suitable line on which to map the angles such that as much information as possible about the directional data is retained.

The generalised projection and geometric variance obtained were shown to have terms that represent dispersion along each orthogonal axis that is needed to represent the directional data. This is a positive feature, which is retained from the $2 \mathrm{D}$ case. In the $3 \mathrm{D}$ case, this feature makes the variances superior to Mardia's spherical variance, which according to the present analysis, can only represent dispersion along one direction. Furthermore, from the point of view of the geometric measure proposed, it was shown that Mardia's spherical variance is equivalent to the mean bias or absolute mean bias, these being measures of dispersion that are usually set aside in favour of the variance. In all cases, it is another positive feature of the geometric measure that it allows a direct derivation for Mardia's spherical variance, which in turn justifies its use as a measure of dispersion.

A look at the generalised projection and geometric variance would reveal that they have the same basic structure. The only real difference between them is that $s_{\mathrm{G} k}^{2}$ emphasises the measure of dispersion along the $x_{1}$-axis more than any other axis by not squaring it and multiplying it by two. Thus, it is not an easy task to discriminate between the two in order to decide which one should be adopted. However, the use of these two measures of dispersion is likely to give researchers a superior tool for modelling angular dispersion in three (and higher) dimensions and hence to obtain a better insight on the way in which pollutants will diffuse.

\section{References}

[1] Mardia, K.V., Statistics of Directional Data, Academic Press: New York, 1972.

[2] Batschelet E., Circular Statistics in Biology, Academic Press: New York, 1981.

[3] Essenwanger, O.M., World Survey of Climatology, Vol. 1, B: General Climatology 1 B: Elements of Statistical Analysis, Elsevier: Amsterdam, 1985.

[4] Irwin, J. S., Dispersion Estimate Suggestion 9: Processing of Wind Data. (document for internal distribution) Environmental Application Branch, Meteorology and Assessment Division, ESRL, U.S. Environmental Protection Agency, Research Triangle Park, N. C., 1980.

[5] Nelson, E. W., A simple and accurate method for calculation of the standard deviation of horizontal wind direction. Journal of the Air Pollution Control Association, 34, pp. 1139-1140, 1984.

[6] Skibin, D., Simple Method for Determining the Standard Deviation of Wind Direction. Journal of Atmospheric and Oceanic Technology, 1, pp. 101-102, 1983. 
[7] Yamartino R.J., A comparison of several "single pass" estimators of the standard deviation of wind direction. Journal of Climate and Applied Meteorology, 23, pp. 1362-1366, 1984.

[8] Farrugia, P. S. \& Micallef, A., Derivation of a new measure of angular dispersion for circular variables using geometrical description. Meteorological Journal, 7(3), pp. 111-117, 2004.

[9] Fisher, N. I., Problems with the Current Definitions of the Standard Deviation of Wind Direction. Journal of Climate and Applied Meteorology, 26, pp. 1522-1529, 1987.

[10] Fisher, N. I., Lewis T. \& Emblet B.J.J., Statistical analysis of spherical data, Cambridge University Press: Cambridge, 1987. 